\title{
Synergistic antimicrobial effect of photodynamic therapy and ciprofloxacin
}

\author{
Maria Rita Ronqui, Tatiana Maria Starck Fogaça de Aguiar Coletti, Laura Marise de Freitas, \\ Elaine Toscano Miranda, Carla Raquel Fontana* \\ Faculdade de Ciencias Farmaceuticas, Univ. Estadual Paulista (UNESP), Rodovia Araraquara-Jau, km 01 - s/n, Campos Ville, Araraquara, Sao Paulo, Brazil
}

\section{A R T I C L E I N F O}

\section{Article history:}

Received 20 March 2015

Received in revised form 25 February 2016

Accepted 26 February 2016

Available online 2 March 2016

\section{Keywords:}

Photodynamic therapy

Methylene blue

Staphylococcus aureus

Escherichia coli

Combined therapy

\begin{abstract}
A B S T R A C T
The occurrence of a variety of pathogens resistant to current antibiotics remains the major problem in medical care, especially when bacterial infections are established as biofilms. In this study, we propose the use of photodynamic therapy (PDT) as a monotherapy and associated with antibiotic as an alternative treatment. The aim of this study was to analyze the effects of PDT mediated by methylene blue (MB) on Staphylococcus aureus (ATCC 25923) and Escherichia coli (ATCC 25922) in both biofilm and planktonic phases. Several concentrations of MB and light doses were tested. The bactericidal effects of PDT as a monotherapy did not increase with the concentration of photosensitizer, but were light dose-dependent. In addition, bacteria in biofilms were less affected than cells in the planktonic phase. Although not concentration-dependent, the disruption effect of PDT on biofilms was clearly illustrated by scanning electron microscopy (SEM). We also carried out experiments that evaluated the synergistic effect of photodynamic therapy and the antibiotic ciprofloxacin. The best results were obtained after combination treatment of photodynamic therapy followed by ciprofloxacin on biofilms, which increased bacterial reduction on biofilms, resulting in a $5.4 \log$ reduction for $S$. aureus biofilm and approximately 7 log for E. coli biofilm.
\end{abstract}

(c) 2016 Elsevier B.V. All rights reserved.

\section{Introduction}

Microorganisms growing in biofilms have been found to be relatively resistant to antimicrobial therapy. The heterogeneous structure composed of bacteria attached which are bound to a surface, surrounded by an extracellular matrix and which display phenotypic traits not observed in their planktonic counterparts can explain how biofilm protects the bacteria within against the host's immune mechanisms and antimicrobial agents. It has been shown that Staphylococcus aureus and Escherichia coli strains are able to adhere to and form biofilms on different biomaterials and medical devices [1].

As an opportunistic pathogen, S. aureus can cause a variety of selflimiting to life-threatening diseases. It is one of the most common causes of skin, soft-tissue, and nosocomial infections. Complications with staphylococcal infection at other sites, or complications of surgical procedures as deep infections include endocarditis, peritonitis, necrotizing pneumonia, bacteremia, meningitis, osteomyelitis, septic arthritis, and infections of bones, joints and organs [2]. E. coli is a well-known member of human intestinal tract that harbors a diverse and complex microbial community, but it is also known to cause infectious endocarditis [3], urinary tract infections [4], bacteremia as well as sepsis [5]. The

\footnotetext{
* Corresponding author.

E-mail addresses: ritaronqui@hotmail.com (M.R. Ronqui), tatistarck@ig.com.br (T.M.S.F. de Aguiar Coletti), lfmarise@gmail.com (L.M. de Freitas), toscae@fcfar.unesp.br (E.T. Miranda), fontanacr@fcfar.unesp.br (C.R. Fontana).
}

biofilm formation of $S$. aureus and $E$. coli strains on surface of synthetic implants may also cause a serious complication as deep surgical site infection encompassing the implants.

Generally, the use of large amounts of antibiotics for long periods is undesirable in clinical practice due to the adverse effects on the microbiota and the increased potential to induce resistance. An effort to improve the therapeutic results and reduce the occurrence of undesirable side effects oriented recent research to search for efficient ways to cause biofilm disturbance via alternative methods of treatment, such as photodynamic therapy (PDT) [5,6].

Shortening, PDT is a treatment based on the interaction of light, photosensitizing agents and oxygen: the photosensitizer molecules are excited by a specific wavelength of light leading to the production of singlet oxygen, highly toxic to cells, culminating in target cell death via oxidative damage. [7]. The main advantages of PDT over conventional antimicrobial therapies include immediate onset of action, elimination of resistant microorganisms and secreted virulence factors, minimized systemic adverse effects, and double selectivity (deleterious effect only on sites where both PS and light are delivered concomitantly).

Photodynamic therapy has been highlighted as an alternative to conventional antimicrobial treatment, either in combination with traditional methods or alone, arising as a non-complex modality with minor side effects [8]. In this study, we aimed to evaluate the effect of PDT individually in suspension and biofilm and its synergistic effect in combination with ciprofloxacin. Associating PDT and ciprofloxacin can bring their individual advantages together, improving bacterial reduction as 
long as reducing photosensitizer and antibiotic concentration and toxicity.

\section{Materials and Methods}

\subsection{Bacterial Inoculum Preparation}

We used American Type Culture Collection reference strains of S. aureus (ATCC 25923) and E. coli (ATCC 25922), provided by the National Institute of Quality Control in Health (INCQS) from the Oswaldo Cruz Foundation (FIOCRUZ), Manguinhos, RJ, Brazil. A single colony of S. aureus or E. coli strains was inoculated into $5 \mathrm{~mL}$ of Tryptic Soy Broth (TSB) and incubated for $24 \mathrm{~h}$ at $37^{\circ} \mathrm{C}$. Afterwards, when the bacterial culture reached the log phase of growth, for adjustment of the inoculum, the absorbance was measured using a spectrophotometer (Biotek ${ }^{\circledR}$, Winooski, VT, USA) with a wavelength of $630 \mathrm{~nm}$. The interval of absorbance reading was considered a range from 0.08 to 0.10 , which corresponds to a $0.5 \mathrm{McF}$ arland scale $\left(\sim 1 \times 10^{8} \mathrm{CFU} / \mathrm{mL}\right)$.

\subsection{Biofilm Development}

Under aerobic conditions, the bacteria were dispersed and added to TSB. For biofilm development, according to the published model [9], 96well plates were previously prepared with $150 \mu \mathrm{L}$ of TSA (Trypticase Soy Agar) or Mueller-Hinton agar. One-hundred and fifty microliters of the inoculum (approximately $10^{8}$ bacteria) was carefully pipetted to fill the agar wells in each 96-well plate. The plates were then incubated aerobically at $35^{\circ} \mathrm{C}$ for five days. After an initial incubation period of $48 \mathrm{~h}$, the liquid medium was carefully aspirated from each well and the biofilms were replenished with fresh broth. Then, fresh TSB was added daily into each well, very slowly, to avoid disruption of the biofilm.

\subsection{Photosensitizer}

Methylene blue (Sigma Co., St. Louis, USA) was dissolved in Phosphate Buffered Saline (PBS) to give solutions at concentrations of 6.25, $12.5,25,50,100,200 \mu \mathrm{g} / \mathrm{mL}$ before use, and $400 \mu \mathrm{g} / \mathrm{mL}$ for biofilm only. The absorption spectra of methylene blue was recorded from 400 to $700 \mathrm{~nm}$ using quartz cuvettes with a 1-cm path length using a Lambda 1050 UV/Vis/NIR spectrophotometer (PerkinElmer, Waltham, MA, USA) and was characterized by a long-wavelength maximum at $665 \mathrm{~nm}$.

\subsection{Light Source}

A compact red light-emitting diode (LED) array-based illumination system at $660 \mathrm{~nm}$ with a homogeneous illumination area composed of 48 LED with variable intensities and a cooling device (IrradLED ${ }^{\circledR}-$ biopdi, São Carlos, SP, Brazil) was used. The distance between the LED and the plate allowed an even distribution of light on each well. The power density of the incident radiation was measured using a power meter (Coherent ${ }^{\circledR}$, Santa Clara, CA, USA). The light energy fluence used in this study for bacterial suspensions were 2.8 and $5.6 \mathrm{~J} / \mathrm{cm}^{2}$, and biofilms were $5.6,11.2$ and $22.4 \mathrm{~J} / \mathrm{cm}^{2}$. The study groups were: 1 . $\mathrm{L}-\mathrm{MB}-$ (control group), 2. L + MB - (treated only with light; light group), 3. $\mathrm{L}-\mathrm{MB}+$ (treated only with methylene blue; $\mathrm{MB}$ group), and $4 . \mathrm{L}+\mathrm{MB}+$ (treated with methylene blue and light; PDT group). Groups 1 and 3 were kept in plates at room temperature and covered with aluminum foil during irradiation of groups 2 and 4 .

\subsection{Planktonic Phase}

Tubes with $1 \mathrm{~mL}$ of inoculum were centrifuged at 13,000 rpm for $15 \mathrm{~min}$ to add $1 \mathrm{~mL}$ of methylene blue for the MB and PDT groups. For the light and control groups, methylene blue was substituted by TSB and samples were homogenized and wrapped in aluminum foil to complete the incubation time (25 min of drug incubation for the MB group and $5 \mathrm{~min}$ for the PDT group). Following incubation, bacterial suspensions were placed in the wells of 96-well plates and exposed to light of $660 \mathrm{~nm}$ at room temperature. During photodynamic therapy, 96-well plates remained covered with a lid, and special care was taken not to disturb the plates. After illumination of the appropriate wells, bacterial suspensions underwent serial dilutions in TSB and $100-\mu \mathrm{L}$ aliquots were plated on TSA plates and then incubated under aerobic conditions for 1 day.

\subsection{Biofilms}

Biofilms were developed as described before. There was one plate for the control and MB groups and other plates for the PDT and LIGHT groups to avoid light exposure of adjacent wells. 96-well plates containing the $S$. aureus biofilms or E. coli biofilm were incubated with TSB (light and control groups) or methylene blue for $5 \mathrm{~min}$ at the PDT group or 25 min at the MB group. Those wells were then simultaneously irradiated with red light.

During photodynamic therapy, 96-well plates remained covered with a lid and were not disturbed. After illumination, adherent bacteria were gently scraped from TSA in each well using a sterile bacteriological loop and dispersed in TSB. The same experienced researcher removed all of the biofilms to ensure that the scrapings collected the entire biofilm and did not add variability to the results. Samples were submitted to serial dilutions and $100-\mu \mathrm{L}$ aliquots were spread over the surface of TSA plates. The plates were incubated aerobically at $37^{\circ} \mathrm{C}$ for 1 day.

\subsection{Minimum Inhibitory Concentration (MIC)}

The ciprofloxacin activity on bacterial growth was determined by the microdilution method. It was conducted based on the Clinical and Laboratory Standards Institute (CLSI, 2006) M7-A6 document for aerobic bacteria [10].

\subsection{Evaluation of Synergism in Planktonic Phase: Ciprofloxacin After PDT}

The MIC plus three sub-inhibitory concentrations (I, II and III) of ciprofloxacin were tested as follows: for $S$. aureus concentrations were $0.5 \mu \mathrm{g} / \mathrm{mL}$ (MIC); $0.25 \mu \mathrm{g} / \mathrm{mL}$ (I); $0.125 \mu \mathrm{g} / \mathrm{mL}$ (II) and $0.0625 \mu \mathrm{g} / \mathrm{mL}$ (III); and for E. coli, $0.004 \mu \mathrm{g} / \mathrm{mL}$ (MIC); $0.002 \mu \mathrm{g} / \mathrm{mL}$ (I); $0.001 \mu \mathrm{g} / \mathrm{mL}$ (II) and $0.0005 \mu \mathrm{g} / \mathrm{mL}$ (III). MB concentrations of $50 ; 25 ; 12.5$ and $6.25 \mu \mathrm{g} / \mathrm{mL}$ were tested and light irradiation was performed at $2.8 \mathrm{~J} / \mathrm{cm}^{2}$; conditions were determined according to the results obtained from PDT assays. After PDT, $100 \mu \mathrm{L}$ of each MB sample was transferred to a microtube containing $100 \mu \mathrm{L}$ of ciprofloxacin; the control group was transferred to a microtube containing $100 \mu \mathrm{L}$ of Mueller-Hinton broth. Samples were then incubated at $37^{\circ} \mathrm{C}$ for $24 \mathrm{~h}$ and, after that, submitted to serial dilution and plated on Mueller-Hinton agar plates, also at $37^{\circ} \mathrm{C}$ for $24 \mathrm{~h}$.

\subsection{Evaluation of Synergism in Planktonic Phase: PDT After Ciprofloxacin}

PDT and antibiotic conditions were the same as described above. Bacterial suspensions were centrifuged at 13,000 rpm for $10 \mathrm{~min}$ and the pellets were suspended in $1 \mathrm{~mL}$ of each ciprofloxacin concentration (MIC, I, II or III). Samples were incubated at $37^{\circ} \mathrm{C}$ for $24 \mathrm{~h}$. Following, PDT was performed as previously described.

\subsection{Evaluation of Synergism in Biofilms: Ciprofloxacin after PDT}

Biofilms were developed in 96-well plates as described above. MB concentrations tested were 200; $100 ; 50$ and $25 \mu \mathrm{g} / \mathrm{mL}$ and energy fluences were 11.2 and $22.4 \mathrm{~J} / \mathrm{cm}^{2}$, determined according to PDT results obtained on biofilms. Ciprofloxacin concentrations tested were all five sub-inhibitory concentrations multiplied for a factor of 1000 (I, II, III, 
IV and V): for S. aureus, de 250 (I); 125 (II); 62.5 (III); 31.25 (IV) and $15.625 \mu \mathrm{g} / \mathrm{mL}$ (V); and for E. coli, 2 (I); 1 (II); 0.5 (III); 0.25 (IV) and $0.125 \mu \mathrm{g} / \mathrm{mL}(\mathrm{V})$. After PDT, $100 \mu \mathrm{L}$ of each MB sample was transferred to a microtube containing $100 \mu \mathrm{L}$ of each ciprofloxacin concentration; the control group was transferred to a microtube containing $100 \mu \mathrm{L}$ of Mueller-Hinton broth. Samples were then incubated at $37^{\circ} \mathrm{C}$ for $24 \mathrm{~h}$ and, after that, submitted to serial dilution and plated on MuellerHinton agar plates, also at $37^{\circ} \mathrm{C}$ for $24 \mathrm{~h}$.

\subsection{Scanning Electron Microscopy}

Bacterial suspensions were standardized from $18 \mathrm{~h}$ culture in Mueller-Hinton broth for S. aureus and E. coli to achieve approximately $1.0 \times 10^{8} \mathrm{CFU} / \mathrm{mL}$. In a $13 \mathrm{~mm}$ diameter glass coverslip, $600 \mu \mathrm{L}$ of the suspension was dispensed and incubated at room temperature for $72 \mathrm{~h}$ for biofilm growth without agitation. After $72 \mathrm{~h}$, the replacement of culture medium was performed and incubated for additional $24 \mathrm{~h}$. Following biofilm formation, samples were submitted to PDT irradiation with $11.2 \mathrm{~J} / \mathrm{cm}^{2}$ and $200 \mu \mathrm{g} / \mathrm{mL}$ of MB. Untreated biofilm was used as control. After treatments coverslips were thoroughly washed with sterile $0.1 \mathrm{M}$ phosphate buffer ( $\mathrm{pH}$ 7.4). The washing procedure was repeated three times and $1 \mathrm{~mL}$ of $3 \%$ glutaraldehyde and $2 \%$ paraformaldehyde in $0.1 \mathrm{M}$ potassium phosphate buffer, $\mathrm{pH} 7.4$ was added. Then, three washes with pure buffer solution were carried out. Dehydration was carried out in increasing concentrations of ethanol (50\%, 60\%, $70 \%$, $80 \%, 90 \%$, and $100 \%$ ) [11,12]. Following dehydration samples were dried in a desiccator with silica for $72 \mathrm{~h}$ and analyzed in a scanning electron microscope (model HITACHI TM3000).

\section{Results}

\subsection{Minimum Inhibitory Concentration}

MIC was measured by determining the optical density (OD) and defined as the lowest concentration of antibiotic allowing no growth after $24 \mathrm{~h}$ of incubation. The MIC of ciprofloxacin for S. aureus was $0.50 \mu \mathrm{g} / \mathrm{mL}$ and $0.004 \mu \mathrm{g} / \mathrm{mL}$ for E. coli.

\subsection{Planktonic Phase}

Methylene blue showed no significant dark toxicity (Figs. 1 and 3) in all concentrations tested. Analyzing the effect of light individually $(\mathrm{L}+\mathrm{MB}-)$, we did not observe significant bacterial reduction either for S. aureus or E. coli at all fluencies tested (Fig. 2).

Either for E. coli or S. aureus PDT was light-dose dependent, given that the same $\mathrm{MB}$ concentrations resulted in no reduction (Fig. $2 \mathrm{~A}$ and B) or more than $5 \log$ reduction (Fig. $2 \mathrm{C}$ and D) for 2.8 or $5.6 \mathrm{~J} / \mathrm{cm}^{2}$,

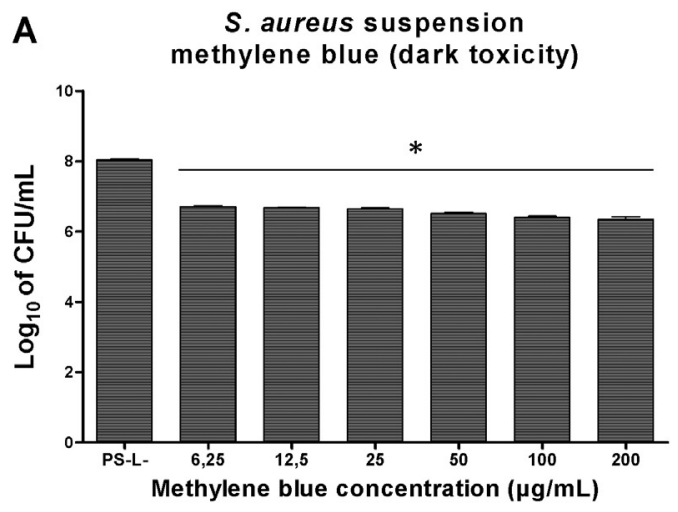

respectively. E. coli was clearly more resistant to MB-PDT than S. aureus, with the best bactericidal effect occurring only at the higher $\mathrm{MB}$ concentration (Fig. 2D).

\subsection{Biofilm Phase}

For both S. aureus and E. coli no significant toxicity was observed for the $\mathrm{MB}$ or the light group (Figs. 3 and 4 ). Since $2.8 \mathrm{~J} / \mathrm{cm}^{2}$ gave no reductions in planktonic assays and biofilms are a more resistant form compared to planktonic cells, light doses used were 5.6 and $11.2 \mathrm{~J} / \mathrm{cm}^{2}$. MB-PDT induced significant killing, also dependent on the light dose: $5.6 \mathrm{~J} / \mathrm{cm}^{2}$ reduced approximately $2.5 \mathrm{log}$ for both $E$. coli and $S$. aureus with all $\mathrm{MB}$ concentrations (Fig. $4 \mathrm{~A}$ and $\mathrm{B}$ ); while the $\mathrm{MB}$ concentration of $200 \mu \mathrm{g} / \mathrm{mL}$ irradiated with $11.2 \mathrm{~J} / \mathrm{cm}^{2}$ increased the reduction to more than $4 \log$ (Fig. 4C and D).

As can be observed, the same MB-PDT parameters led to more than $5 \log$ reduction of planktonic cells, but only $2.5 \mathrm{log}$ reduction of biofilms, proving once more that biofilm is an effective resistance state for bacteria.

\subsection{Combined Effect of PDT and Ciprofloxacin}

\subsubsection{Planktonic Phase}

We sought to investigate if PDT combined with ciprofloxacin (CIP) would have a synergistic effect on the bacterial reduction, and also if the treatment order (PDT + antibiotic, or antibiotic + PDT) would result in different outcomes. Sub-inhibitory parameters were chosen, so the possible synergistic effect would be prominent. Results are described below.

3.4.1.1. Ciprofloxacin Followed by PDT. For S. aureus (Fig. 5A), even with the lowest CIP and MB concentrations $(0.0625$ and $6.25 \mu \mathrm{g} / \mathrm{mL}$, respectively), bacterial killing was significantly increased when compared to MB-PDT alone for the same light dose (Fig. 2A), resulting in approximately $2 \log$ reduction. Augmenting ciprofloxacin concentration led to proportional killing, resulting in approximately $5 \log$ reduction with $0.5 \mu \mathrm{g} / \mathrm{mL}$ of CIP and $50 \mu \mathrm{g} / \mathrm{mL}$ of MB. E. coli, on the other hand, was significantly reduced $(\sim 4 \log )$ only at the highest CIP concentration (Fig. 5B). Comparing these results with those obtained with MB-PDT at $2.8 \mathrm{~J} / \mathrm{cm}^{2}$, combining the therapies led to a significantly better outcome. (See Fig. 6.)

3.4.1.2. PDT Followed by Ciprofloxacin. For S. aureus (Fig. 5C), also with the lowest CIP and MB concentrations the bacterial killing was significantly increased when compared to MB-PDT alone for the same light dose (Fig. 2A), also resulting in approximately 2 log reduction. Augmenting $\mathrm{MB}$ and $\mathrm{CIP}$ concentrations resulted in increased reductions,

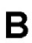

B

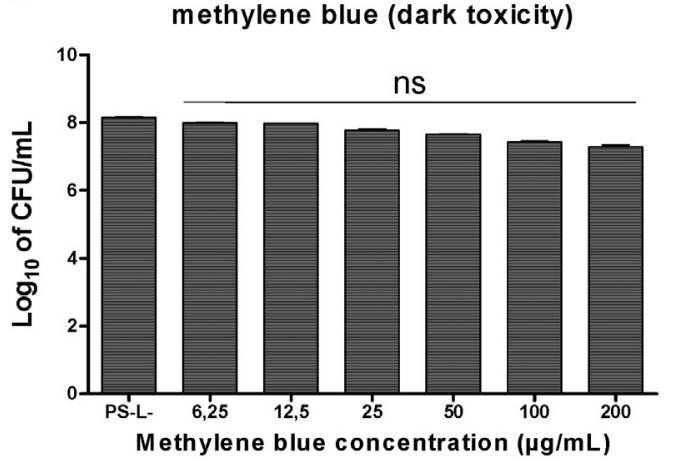

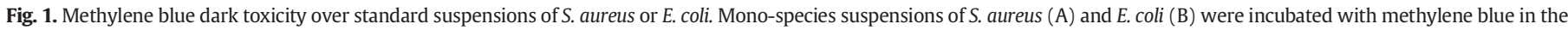

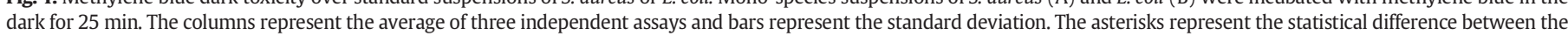
groups and the control (one-way ANOVA with Tukey's posthoc). ${ }^{*} \mathrm{p}<0.05$; ${ }^{* *} \mathrm{p}<0.01$; ${ }^{* * *} \mathrm{p}<0.001$; ns: not significant. PS: photosensitizer; L: light. 
A

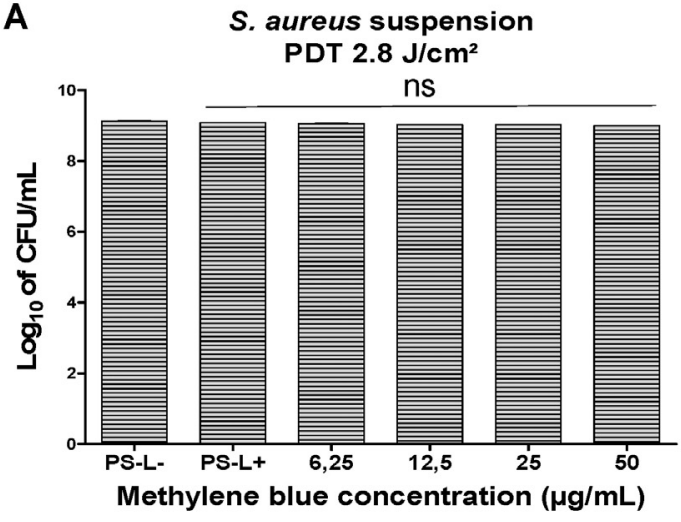

C
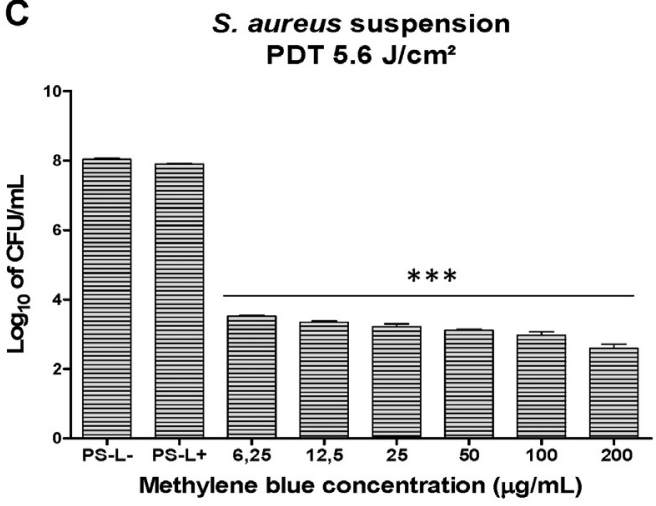

B

E. coli suspension

PDT $2.8 \mathrm{~J} / \mathrm{cm}^{2}$

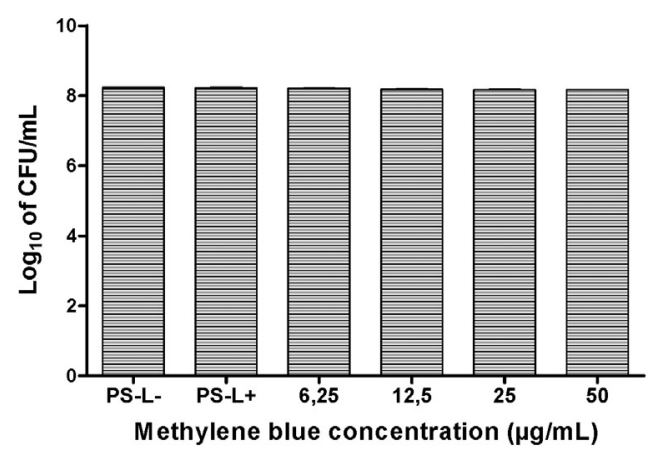

D

\section{E. coli suspension PDT $5.6 \mathrm{~J} / \mathrm{cm}^{2}$}

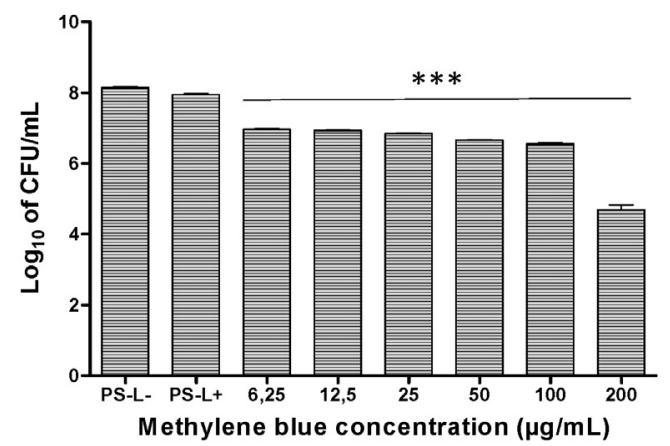

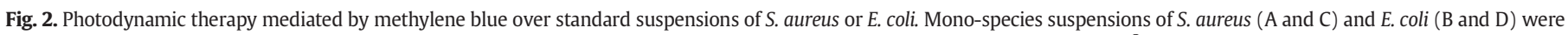

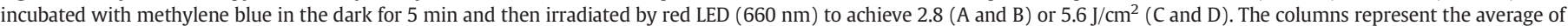

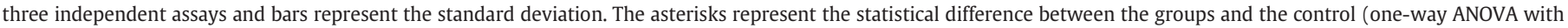
Tukey's posthoc). ${ }^{*} \mathrm{p}<0.05 ;{ }^{* *} \mathrm{p}<0.01 ;{ }^{* * *} \mathrm{p}<0.001$; ns: not significant. PS: photosensitizer; L: light.

achieving more than 6 log for some combinations (Fig. 2C). Under the same parameters of MB-PDT, E. coli reduction was clearly dependent upon ciprofloxacin concentration (Fig. 5D), with no significant killing at the two lowest concentrations of the antibiotic, but more than $4 \log$ reduction at the two highest, regardless of $\mathrm{MB}$ concentration. Compared to MB-PDT alone (Fig. 2B), as previously stated for S. aureus, the combination of ciprofloxacin and PDT resulted in an improved killing effect.

Overall, the order of the treatments did not affect the results over S. aureus, except for MB concentrations of 25 and $50 \mu \mathrm{g} / \mathrm{mL}$, which resulted in improved killing when PDT preceded the antibiotic. E. coli reductions, on the other hand, were more affected, ranging from barely no reduction when antibiotic preceded PDT to more than 4 log reduction when PDT preceded the antibiotic.

\subsubsection{Biofilm Phase}

Given that PDT preceding the antibiotic had better results on planktonic phase assays, biofilms were treated only with that order of combination. Light doses chosen were 11.2 , based on planktonic assays, and $22.4 \mathrm{~J} / \mathrm{cm}^{2}$, in an attempt of maximizing the combination effect. Also, CIP concentrations were increased by a factor of 1000 , according to literature data [13].
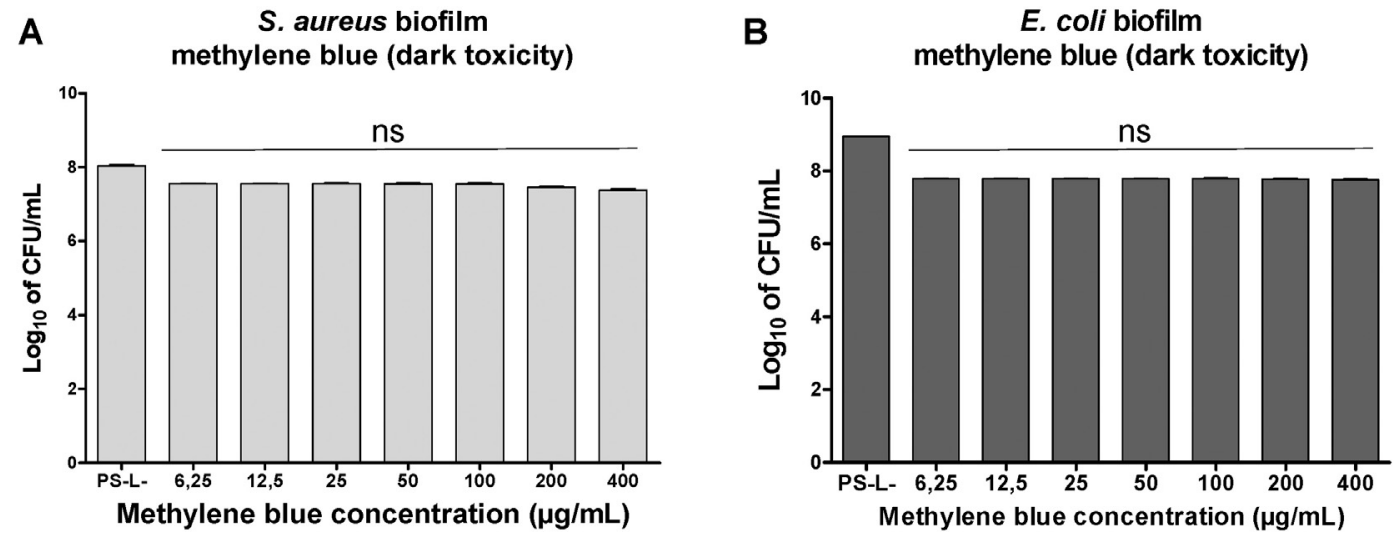

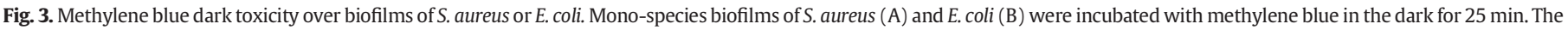

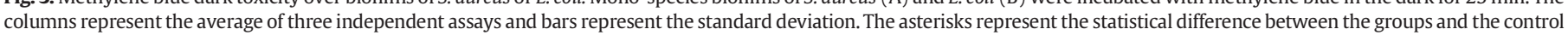
(one-way ANOVA with Tukey's posthoc). ${ }^{*} \mathrm{p}<0.05 ;{ }^{* *} \mathrm{p}<0.01{ }^{* * *} \mathrm{p}<0.001$; ns: not significant. PS: photosensitizer; L: light. 
A

$$
\begin{aligned}
& \text { S. aureus biofilm } \\
& \text { PDT } 5.6 \mathrm{~J} / \mathrm{cm}^{2}
\end{aligned}
$$

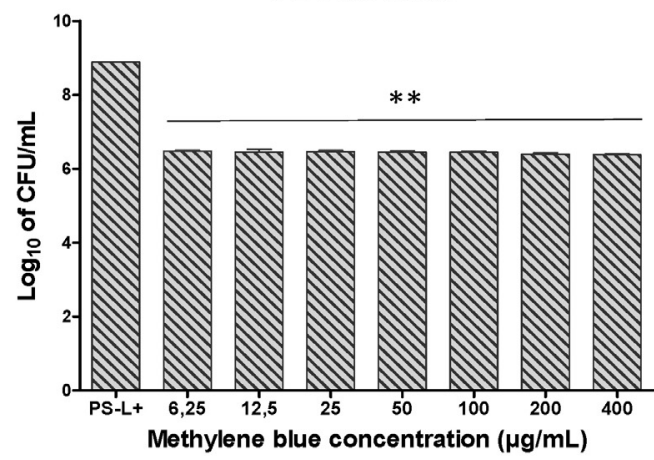

C

\section{S. aureus biofilm PDT $11.2 \mathrm{~J} / \mathrm{cm}^{2}$}

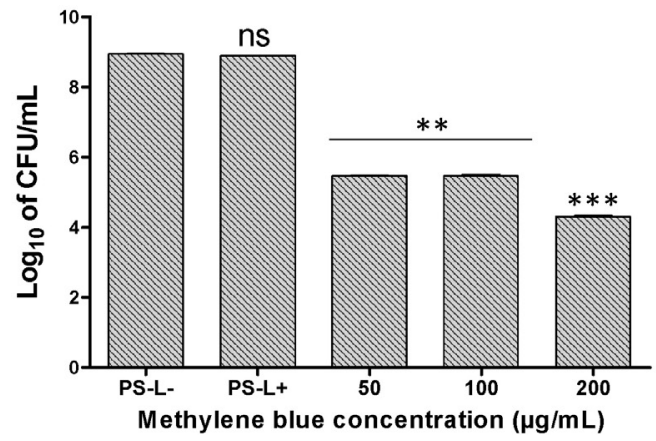

B

E. coli biofilm PDT $5.6 \mathrm{~J} / \mathrm{cm}^{2}$

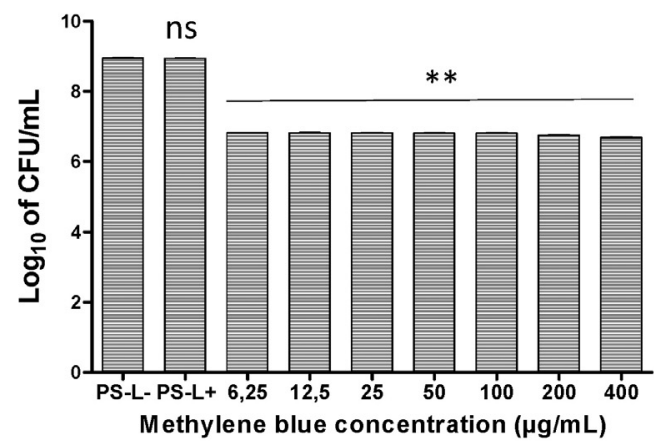

D

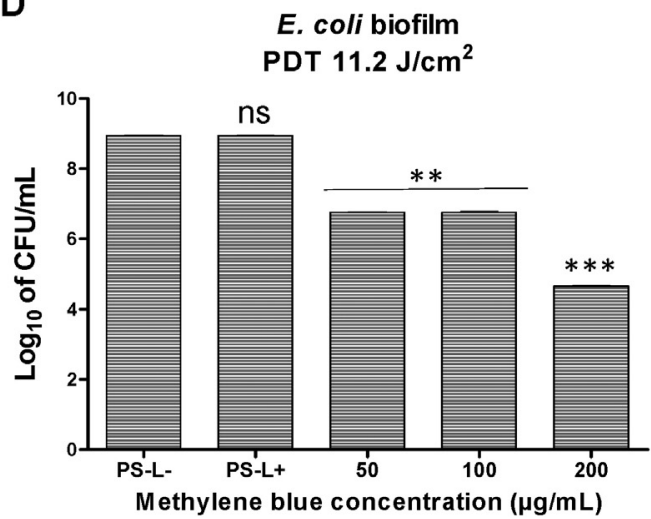

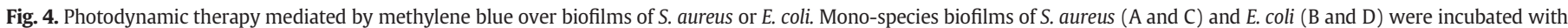

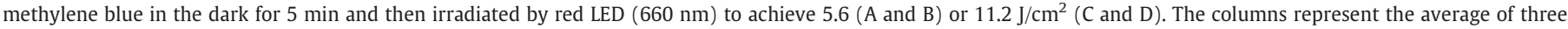

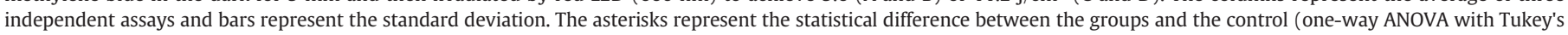
posthoc). ${ }^{*} \mathrm{p}<0.05 ;{ }^{* *} \mathrm{p}<0.01 ;{ }^{* * *} \mathrm{p}<0.001$; ns: not significant. PS: photosensitizer; L: light.

Both S. aureus and E. coli had similar results, with no changing on outcomes when light dose increased, implying that light dose was not a limiting parameter in that case. Compared to MB-PDT at $11.2 \mathrm{~J} / \mathrm{cm}^{2}$ as a monotherapy (Fig. 4C), the combination of PDT and CIP had an improvement of almost $1 \log$ in $S$. aureus and of approximately $2.4 \log$ in E. coli biofilm reduction.

\subsection{Scanning Electron Microscopy (SEM)}

SEM showed a disruption of biofilm structure and a reduction in the cell number when samples were treated with PDT. PDT group biofilms were dispersed, with the development of several micro-colonies, and not a dense and compact biofilm as observed for the control group (Figs. 7 and 8), proving once more PDT's potential as an alternative treatment to reduce microbial biofilms.

\section{Discussion}

The development of strategies to combat bacteria growing in biofilms is a challenging task, given that those bacteria are much more resistant to antimicrobial therapies. Photodynamic therapy (PDT) may be a promising therapeutic option to effectively control the growth of microbial biofilms. However, as with other antimicrobial therapies, PDT is generally less effective against microorganisms growing in biofilms than against planktonic cells. Thus, a need exists to develop a therapeutic approach that would increase the sensitivity of microorganism to established methods, combining the various modes of antimicrobial action to achieve a synergistic effect.

One benefit of combination therapies is that they may reduce the development of drug resistance, since a pathogen is less likely to have resistance to multiple treatments simultaneously [14]. Another advantage is the possibility to use lower concentration of drugs in combination, diminishing side effects and toxicity [15]. In the present study we evaluated the effects of photodynamic therapy with methylene blue as a monotherapy and combined with ciprofloxacin. Different protocols have been tested and the effect of order of treatments (antibiotic after PDT or PDT after antibiotic) was also evaluated.

The literature shows numerous photosensitizers (PSs) with promising PDT properties for PDT proposes. One of the traditional PSs that has been used for a diversity of applications is methylene blue (MB) [16]. This dye has been approved by the United States Food and Drug Administration (FDA) for treatment of methemoglobinemia in a nonphotodynamic mode. However, when activated, MB produces a high quantum yield of singlet oxygen in the therapeutic window (600 to $800 \mathrm{~nm}$ ). When coupled with its inherently low toxicity, $\mathrm{MB}$ is a promising candidate for use as a PDT agent [17]. Variations of methylene blue concentrations from 6.25 to $200 \mu \mathrm{g} / \mathrm{mL}$ were tested in this study. Dark toxicity evaluation was conducted in the absence of irradiation. For E. coli and S. aureus, treatment with MB alone was not bactericidal at any concentration tested.

Our results related to the irradiation with the LED only showed no reduction in the CFU when the irradiation parameters used were $2.8 \mathrm{~J} / \mathrm{cm}^{2}$ and $5.6 \mathrm{~J} / \mathrm{cm}^{2}$. Experiments have shown that for $S$. aureus and E. coli growth inhibition was not observed with exposure to red LED light, which is consistent with our results [1].

Caring out photodynamic therapy, we observed that all MB concentrations resulted in significant reductions in $S$. aureus suspension, from 4.53 to $5.45 \mathrm{log}$, when submitted to $5.6 \mathrm{~J} / \mathrm{cm}^{2}$ of light. For E. coli, the highest concentration tested presented a reduction of $3.46 \log$. Such bacterial reductions demonstrate that the gram-positive strain was more sensitive to PDT than the gram-negative, an outcome consistent with the literature [18,19]. Gram-negative strains, like E. coli, are intrinsically more resistant than gram-positives to antimicrobial agents due 
A

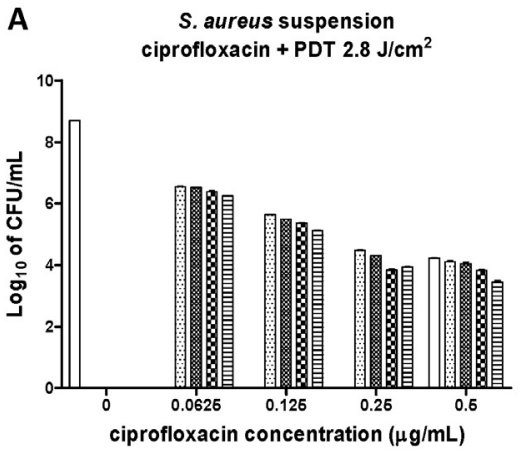

C

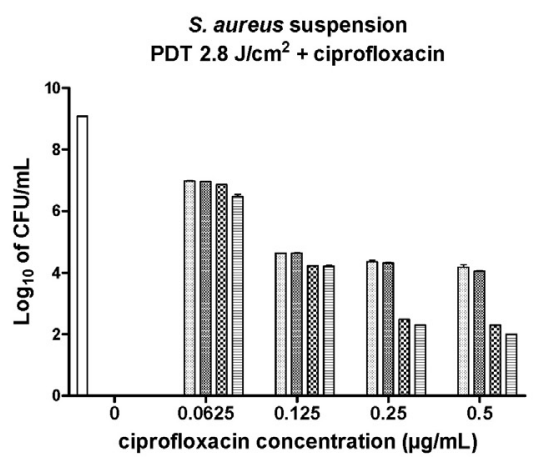

B
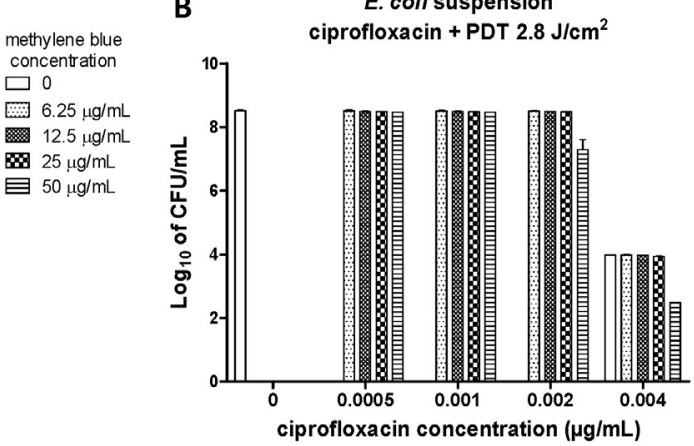

D

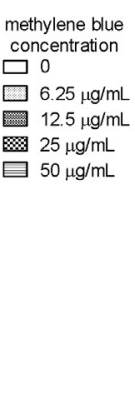

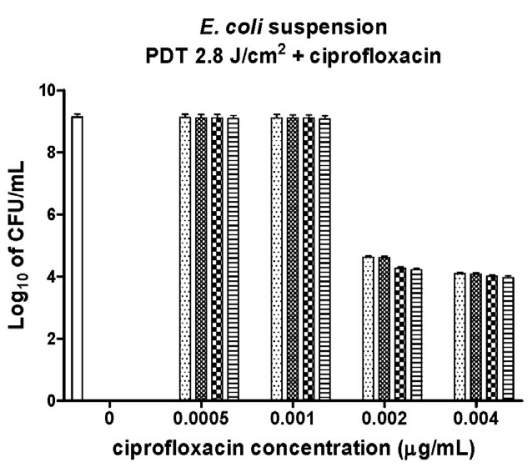

methylene blue

concentration

$\square 0$

$6.25 \mu \mathrm{g} / \mathrm{mL}$

$12.5 \mu \mathrm{g} / \mathrm{mL}$

$825 \mathrm{\mu g} / \mathrm{mL}$

$50 \mu \mathrm{g} / \mathrm{mL}$

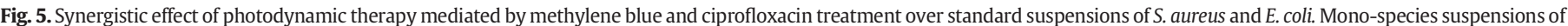

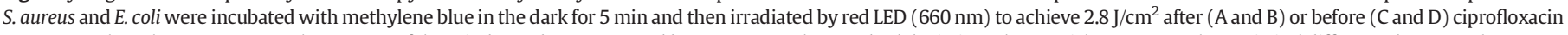

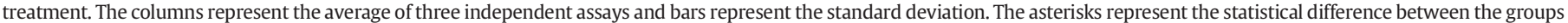
and the control (one-way ANOVA with Tukey's posthoc). ${ }^{*} \mathrm{p}<0.05$; ${ }^{* *} \mathrm{p}<0.01$; ${ }^{* * *} \mathrm{p}<0.001$; ns: not significant. PS: photosensitizer; L: light.

to the more selective nature of their cell envelope [20,21]. In addition, a plethora of studies have shown that PDT is an effective antimicrobial treatment to eliminate $S$. aureus, including the resistant strains such as MRSA [2], again endorsing our results.

To improve the bacterial reduction, the synergist effect of associating PDT with ciprofloxacin was analyzed. Our results show that such association can offer several advantages, especially when PDT precedes the antibiotic. In that case, sub-inhibitory concentrations (below MIC) of ciprofloxacin and lower concentrations of MB combined with lower fluencies may be used to yield the highest bacterial reductions. Applying PDT first may cause enough cell wall damage to increase the uptake of the antibiotic, intensifying the overall antibacterial effect.

In summary, for planktonic experiments the efficiency of bacterial inactivation via PDT is evident and even more satisfactory when PDT is combined with an antibiotic, proving that the synergistic effect of those individual therapies can be a novel treatment approach. Furthermore, a recent study by Vadekeetil and co-workers [14] demonstrated that ciprofloxacin, besides its bacteriostatic activity, exhibits an antivirulence effect when administrated at low concentrations, which highlights its potential as an adjuvant drug in combination therapies.

For biofilm assays, the MB group at a maximum of $400 \mu \mathrm{g} / \mathrm{mL}$ and the light group at a maximum of $22.4 \mathrm{~J} / \mathrm{cm}^{2}$ did not show any bacterial reduction, as expected. A recent study shows that LED irradiation at $30 \mathrm{~J} / \mathrm{cm}^{2}$ and $60 \mathrm{~J} / \mathrm{cm}^{2}$ did not cause bacterial killing, consistent with our findings. [22].

S. aureus and $E$ coli biofilms showed resistance to PDT, with no killing at fluence of $5.6 \mathrm{~J} / \mathrm{cm}^{2}$ at all $\mathrm{MB}$ concentrations. Increasing fluence to $11.2 \mathrm{~J} / \mathrm{cm}^{2}$ the reduction obtained for $S$. aureus was $4.65 \mathrm{log}$ and 4.3 $\log$ for E. coli, both with MB at $200 \mu \mathrm{g} / \mathrm{mL}$. Incomplete bacterial killing by photodynamic therapy is not limited to methylene blue. In a reported study [23], a conjugate of chlorine6 and poly-L-lysine failed to eradicate microorganisms completely in dental plaque scrapings.
The better reductions obtained by increasing energy fluence are due to a greater input of photons being delivered at the target region, which allows MB molecules to interact more intensively with the surroundings and, consequently, generate a major ROS cascade leading to a greater bactericidal effect [24].

The ability of many bacteria to form biofilms provides an important virulence factor. The bacteria surrounded by a biofilm are more difficult to be reduced than those in the planktonic form, and once a biofilm is established it becomes a source of contamination for products and surfaces. In vitro studies indicated that bacterial strains growing in biofilms might become 10-1000 times more resistant to the effects of sanitizers than the same strain in planktonic form. Previous studies have shown that $E$. coli biofilms are more resistant to the antibiotic ciprofloxacin than its planktonic phase. The MIC found to biofilms was 1000 times higher than planktonic phase MIC of ciprofloxacin, or ever higher. Moreover, biofilms are capable of releasing planktonic cells from the outer layers, enabling persistent bacterial infection [25].

Regardless of the increased resistance of bacteria involved in the biofilm, the association of PDT and ciprofloxacin improved biofilm bacteria killing. Our results show that the combination of photodynamic therapy with ciprofloxacin was more effective than both the antibiotic and PDT as monotherapies in reducing the biofilm.

PDT as a monotherapy for S. aureus (at $11.2 \mathrm{~J} / \mathrm{cm}^{2}$ and $200 \mu \mathrm{g} / \mathrm{mL}$ of $\mathrm{MB}$ ) resulted in a reduction of $4.65 \mathrm{log}$. On the other hand, synergistic effect of that therapy with $62.5 \mu \mathrm{g} / \mathrm{mL}$ of ciprofloxacin led to a reduction of $5.24 \log$. For E. coli, PDT with the same parameters promoted a reduction of $4.3 \mathrm{log}$; by combining PDT with CIP the reductions obtained were close to $7 \log$.

A previous study showed that pre-treatment of $S$. aureus biofilms with PDT followed by vancomycin application in concentrations lower than the MIC led to a biofilm matrix disruption, allowing for the killing of bacteria almost entirely [25]. Other studies also investigated the effect of PDT on the susceptibility of S. aureus and Staphylococcus epidermidis to 
A

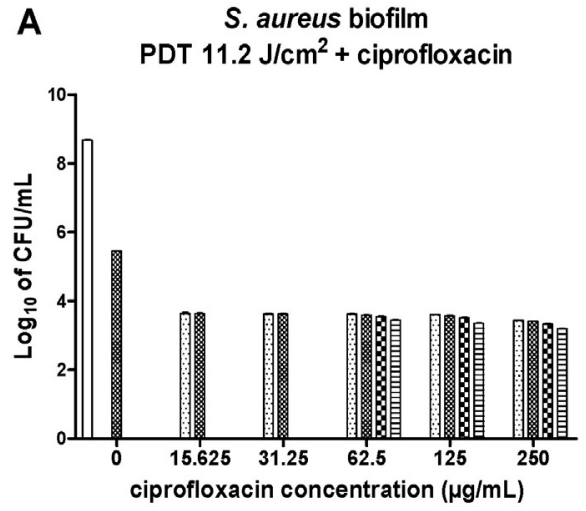

C

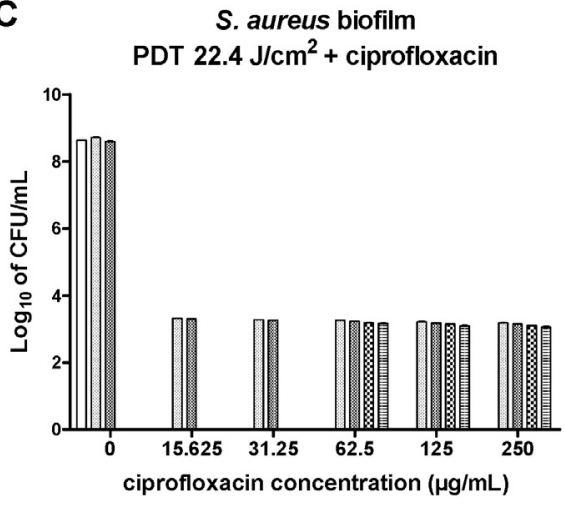

B

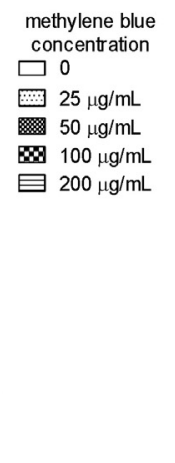

D

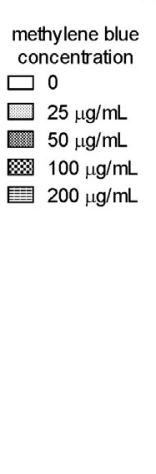

E. coli biofilm

PDT $11.2 \mathrm{~J} / \mathrm{cm}^{2}+$ ciprofloxacin

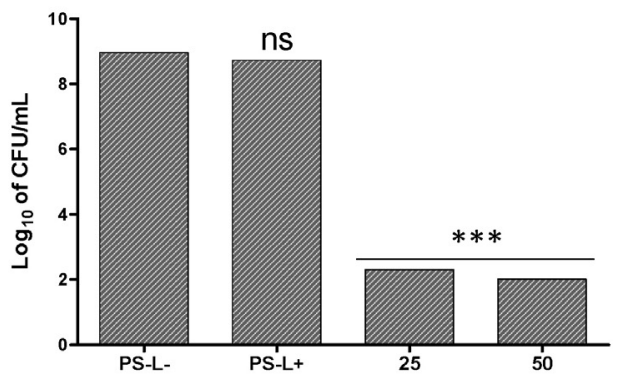

Methylene blue concentration $(\mu \mathrm{g} / \mathrm{mL})$

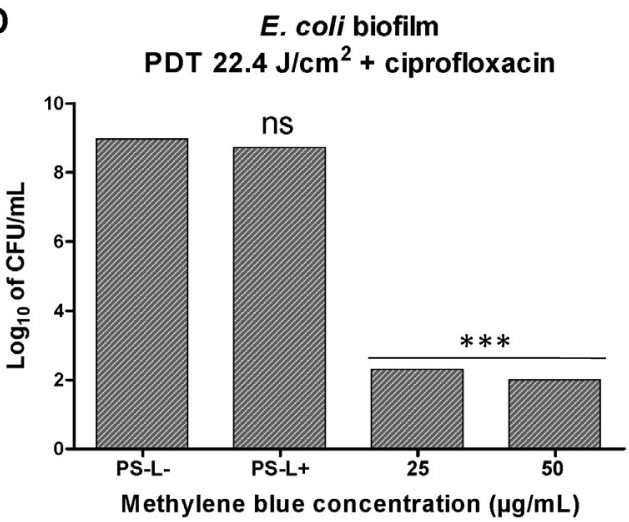

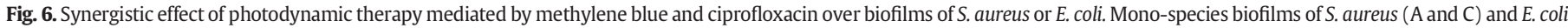

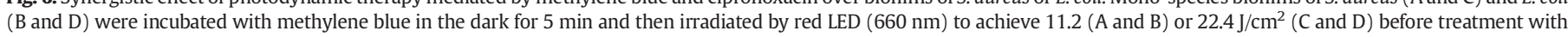

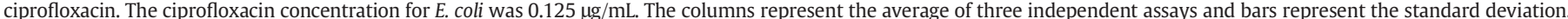

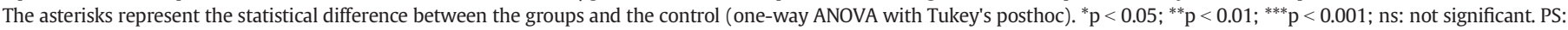
photosensitizer; L: light.

vancomycin, showing a synergistic effect between PDT and antibiotic treatment. Both studies showed that PDT increased susceptibility to vancomycin due to the PDT-induced disruption of biofilm protective matrix that involves bacterial cells, making them susceptible to the antibiotic $[25,26]$, supporting our results and emphasizing the role of PDT and antibiotic combination as a strategy to improve the therapy outcomes over biofilms.

Biofilm morphological changes induced by PDT in S. aureus and E. coli were also observed. The SEM images showed that the PDT promoted morphological changes on both gram-positive and gramnegative bacterial biofilms. For S. aureus, PDT promoted loss of contact between the biofilm mass with scattering and impaired aggregation. For E. coli, after PDT the confluence was amended by disruption of dense and homogeneous structure (mat structure) observed in the control group. Taking together, those results indicate that PDT enhances biofilm sensitivity to antimicrobial treatments by disrupting the extracellular matrix and "turning" biofilm cells into planktonic cells.
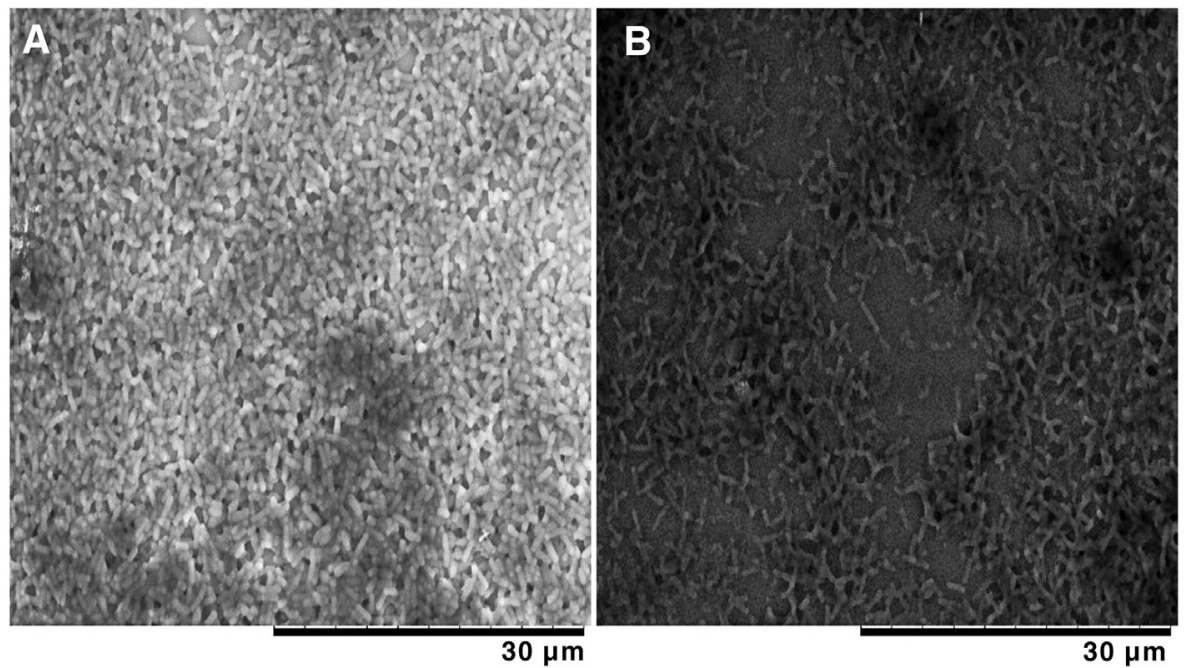

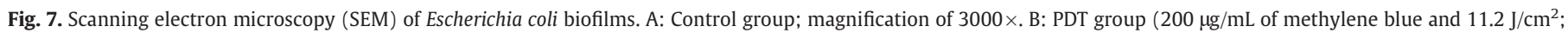
magnification of $3000 \times$. Scale bar: $30 \mu \mathrm{m}$. 

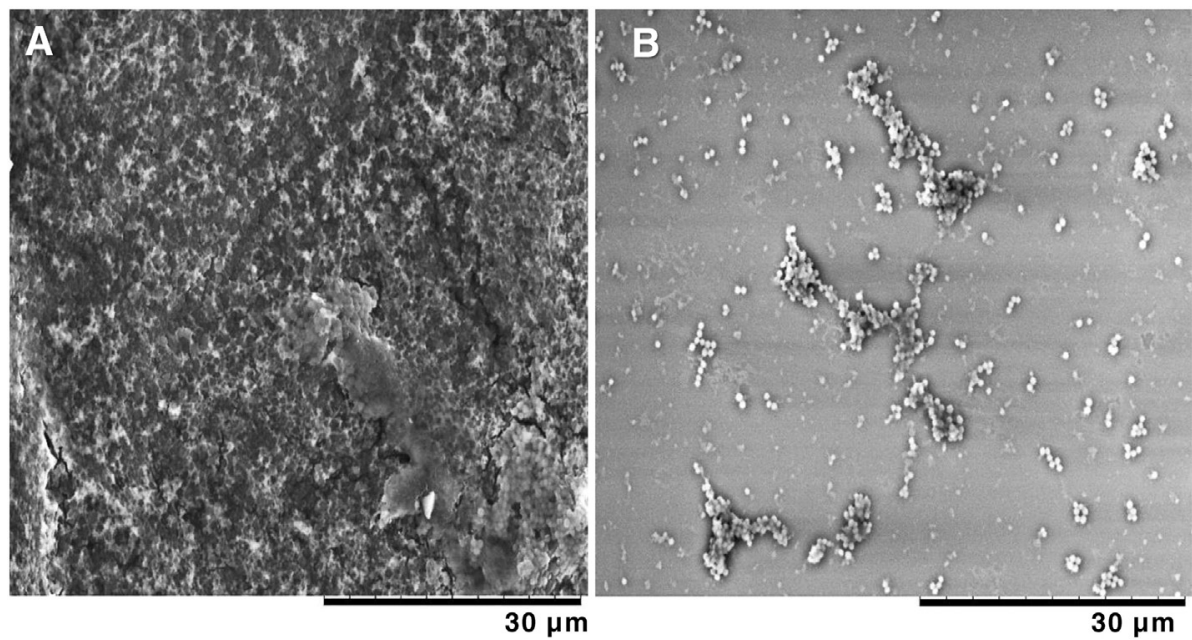

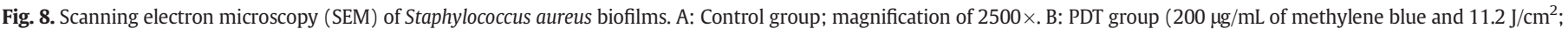
magnification of $2500 \times$. Scale bar: $30 \mu \mathrm{m}$.

\section{Conclusion}

Regarding the efficiency of bacterial inactivation, gram-positive bacteria were more susceptible to PDT than gram-negative species. We observed that the PDT groups presented bacterial reductions higher than the other groups tested. For planktonic phase, when the antibiotic is applied after PDT lower concentrations of ciprofloxacin (lower than the MIC) could be used, since the first sub-inhibitory concentration led to bacterial reduction both to $S$. aureus and $E$. coli. Biofilm bacteria showed resistance to photodynamic therapy; however, the synergistic effect of combined therapy (PDT + ciprofloxacin) overcame that inconvenience. On scanning electron microscopy of biofilms, we could observe that PDT-treated samples were sparse and some of them presented only micro-colonies and not a dense and compact biofilm as observed for control group samples, confirming the disruptive effect of PDT over bacterial biofilms.

\section{Author's Disclosure of Potential Conflicts of Interest}

The authors indicate that there are no potential conflicts of interest.

\section{Acknowledgments}

Financial support from FAPESP 2012/21203-4 (Fundação de Amparo a Pesquisa do Estado de São Paulo), CAPES and CNPq is gratefully acknowledged. We also thank Prof. Dr. Cleber Renato Mendonça (Photonics Group - Institute of Physics - University of São Paulo - IFSC, USP) for scanning electron microscopy analyses.

\section{References}

[1] F. Cieplik, L. Tabenski, W. Buchalla, T. Maisch, Antimicrobial photodynamic therapy for inactivation of biofilms formed by oral key pathogens, Front. Microbiol. 5 (2014) 1-17, http://dx.doi.org/10.3389/fmicb.2014.00405.

[2] T. Agrawal, et al., Harnessing the power of light to treat staphylococcal infections focusing on MRSA, Curr. Pharm. Des. 21 (2015) 1-13, http://dx.doi.org/10.2174/ 1381612821666150310102318.

[3] T.K. Lauridsen, et al., Infectious endocarditis caused by Escherichia coli, Scand. J. Infect. Dis. 43 (2011) 545-546, http://dx.doi.org/10.3109/00365548.2011.554432.

[4] S.K. Chang, Dy Lo, H.W. Wei, H.C. Kuo, Antimicrobial resistance of Escherichia coli isolates from canine urinary tract infections, J. Vet. Med. Sci. 77 (1) (2015) 59-65 (doi: 10.1292/jvms.13-0281)

[5] L.P. Rosa, et al., Effectiveness of antimicrobial photodynamic therapy using a $660 \mathrm{~nm}$ laser and methyline blue dye for inactivating Staphylococcus aureus biofilms in compact and cancellous bones: an in vitro study, Photodiagn. Photodyn. Ther. 12 (2015) 276-281, http://dx.doi.org/10.1016/j.pdpdt.2015.01.001.

[6] M. Tim, Strategies to optimize photosensitizers for photodynamic inactivation of bacteria, J. Photochem. Photobiol. B Biol. 150 (2015) 2-10, http://dx.doi.org/10. 1016/j.jphotobiol.2015.05.010
[7] P. Mroz, et al., Stimulation of anti-tumor immunity by photodynamic therapy, Expert. Rev. Clin. Immunol. 7 (1) (2011) 75-91.

[8] T. Dai, Y.-Y. Huang, M.R. Hamblin, Photodynamic therapy for localized infections state of the art, Photodiagn. Photodyn. Ther. 6 (617) (2009) 170-188.

[9] C.R. Fontana, et al., The antibacterial effect of photodynamic therapy in dental plaque-derived biofilms, J. Periodontal Res. 44 (6) (2009) 751-759.

[10] CLSI, Manual Clinical and Laboratory Standards Institute. Methods for dilution antimicrobial susceptibility tests for bacteria that grow aerobically; approved standards -6 th ed, Document M7-A6 performance standards for antimicrobial susceptibility testing, Clinical and Laboratory Standards Institute, Wayne, PA., 2006

[11] S.J.B. Reed, Electron Microprobe Analysis and Scanning Electron Microscopy in Geology, Cambridge University Press, New York, 1996 (201 pp.).

[12] G. Ramage, K.V. Walle, B.L. Wickes, J.L. López-Ribot, Standardized method for in vitro antifungal susceptibility testing of Candida albicans biofilms, Antimicrob. Agents Chemother. 45 (2001) 2475-2479.

[13] H. Ceri, M.E. Olson, C. Stremick, R.R. Read, D. Morck, A. Buret, The Calgary biofilm device: new technology for rapid determination of antibiotic susceptibilities of bacterial biofilms, J. Clin. Microbiol. 37 (6) (1999) 1771-1776.

[14] A. Vadekeetil, V. Alexandar, S. Chhibber, K. Harjai, Adjuvant effect of cranberry proanthocyanidin active fraction on antivirulent property of ciprofloxacin against Pseudomonas aeruginosa, Microb. Pathog. (2015), http://dx.doi.org/10.1016/j. micpath.2015.11.024.

[15] A. Taraszkiewicz, F. Grzegorz, G. Mariusz, J. Nakonieczna, Innovative strategies to overcome biofilm resistance, Biomed. Res. Int. 2013 (2012) 1-13.

[16] D.E. Gabrielli, D. Belisle, A.J. Severino, M.S. Kowaltowski, Binding, aggregation, and photochemical properties of methylene blue in mitochondrial suspensions, Photochem. Photobiol. 79 (2004) 227-232.

[17] J. Wu, H. Xu, W. Tang, R. Kopelman, M.A. Philbert, C. Xi, Eradication of bacteria in suspension and biofilms using methylene blue-loaded dynamic nanoplatforms, Antimicrob. Agents Chemother. 53 (7) (2009) 3042-3048, http://dx.doi.org/10. 1128/AAC.01604-08.

[18] O'riordan K, Akilov OE, Hasan T. The potential for photodynamic therapy in the treatment of localized infections. Photodiagn. Photodyn. Ther. 2, 4, p. 247-226. http://dx.doi.org/10.1016/S1572-1000(05)00099-2.

[19] T.N. Demidova, M.R. Hamblin, Photodynamic therapy targeted to pathogens, Int. J. Immunopathol. Pharmacol. 17 (3) (2004) 245-254.

[20] R.M. Epand, et al., Molecular mechanisms of membrane targeting antibiotics, Biochim. Biophys. Acta (2015), http://dx.doi.org/10.1016/j.bbamem.2015.10.018.

[21] N. Malanovic, K. Lohner, Gram-positive bacterial cell envelopes: the impact on the activity of antimicrobial peptides, Biochim. Biophys. Acta (2015), http://dx.doi.org/ 10.1016/j.bbamem.2015.11.004.

[22] T.S. Mang, D.P. Tayal, R. Baier, Photodynamic therapy as an alternative treatment for disinfection of bacteria in oral biofilms, Lasers Surg. Med. 44 (7) (2012) 588-596, http://dx.doi.org/10.1002/lsm.22050.

[23] N.S. Soukos, S.E. Mulholland, S.S. Socransky, A.G. Doukas, Photodestruction of human dental plaque bacteria: enhancement of the photodynamic effect by photomechanical waves in an oral biofilm model, Lasers Surg. Med. 33 (2003) 161-168.

[24] J.S.M. Giusti, et al., Antimicrobial photodynamic action on dentin using a lightemitting diode light source, Photomed. Laser Surg. 26 (4) (2008) 281-287.

[25] A. Di Poto, et al., The effect of photodynamic treatment combined with antibiotic action or host defense mechanisms on, Staphylococcus aureusbiofilms, Biomaterials 30 (18) (2009) 3158-3166.

[26] M.S. Sbarra, et al., The photodynamic effect of tetra-substituted N-methyl-pyridylporphine combined with the action of vancomycin or host defense mechanisms disrupts Staphylococcus epidermidis biofilms, Int. J. Artif. Organs 32 (9) (2009) 574-583. 